tolerance in children. We performed a systematic search of the databases PubMed, Pediatrics Pulmonology, European journal of paediatrics, chest physician and Cochrane review for last 5 years.

Method Thirteen clinical studies and Randomized control trials which were done on children treated with hypertonic saline with bronchiolitis hospitalised in an emergency department and general ward were included from year 2014-2019. We included 8 RCT, 1 meta-analysis, 1 Cochrane or 1 decision analysis and 2 Trial sequential analysis and excluded the studies/trials included in Cochrane review 2017 and Meta-analysis 2018. Main outcome of 8 trial out of these 13 trials was 'Length of stay(LOS)' in hospital and 'admission rate' was studied in 1 of these. 1 trial used 'fit for discharge' as main outcome in their trial and 2 Trials looked at the cost effectiveness of use of HS in bronchiolitis.

Results The available studies suggest that Hypertonic saline is promising and safe to use in children with bronchiolitis but there is no superiority found when compared with NS or standard care in reducing the length of hospital stay or admission from emergency department.

Conclusion Hypertonic saline is safe to use in children with bronchiolitis but there is no extra benefit of it in reducing the disease severity, admissions rate or length of stay.

\section{G298(P) DIAGNOSTIC DILEMMA: RESPIRATORY DISTRESS IN A PRE-TERM WITH SITUS INVERSUS TOTALIS}

MN Naseem, MA Alam. Newham Neonatal Unit, Barts Health NHS Trust, London, UK

\subsection{6/archdischild-2020-rcpch.259}

Introduction Primary ciliary dyskinesia (PCD) is a rare inherited disease affecting motile cilia lining the respiratory tract. ${ }^{1}$ Despite neonatal respiratory distress as an early feature, diagnosis is typically delayed until late childhood.

Case Summary We herein report a case of a late pre-term neonate born at 35 weeks and 2 days with respiratory distress from birth. The baby had to be intubated for a day, shifted between BiPAP and CPAP over 7 days before weaning down to nasal canula oxygen and eventually self-ventilating in air by 2 weeks. He has situs inversus with dextrocardia (situs inversus totalis). He also had an episode of right lung collapse which re-expanded on its own. There is family history of consanguinity and 2 previous neonatal deaths for which the parents were investigated extensively by clinical geneticist but without any conclusive etiology. Baby is referred to Royal Brompton Hospital to be investigated for primary ciliary dyskinesia in an outpatient setting.

Aim The purpose of this case report is to include the possibility of primary ciliary dyskinesia as an earlier diagnosis to facilitate earlier onset of therapy, which would help reduce longterm pulmonary morbidity and mortality. ${ }^{2}$

Results The patient was diagnosed with primary ciliary dyskinesia with cilia completely static on light microscopy and immunofluorescent antibody staining showed an absence of the outer dynein arms.

Conclusion Primary ciliary dyskinesia should be part of differential diagnosis of a neonate who has respiratory distress syndrome in the neonatal period with background history of situs totalis inversus in order to decrease long term pulmonary morbidity and to increase quality of life for the infant.

\section{REFERENCES}

1. Tara Mullowney, David Manson, Raymond Kim, Derek Stephens, Vibhuti Shah, Sharon Dell: Pediatrics. 2014 Dec; 134(6): 1160-1166. doi: 10.1542/peds.20140808

2. Holzmann, D. \& Felix, H. Eur J Pediatr (2000) 159: 857. https://doi.org/10.1007/ PL00008354

\section{G299(P) ANTIBIOTIC USAGE AND CHEST RADIOGRAPHY IN CHILDREN ADMITTED FOR COMMUNITY ACQUIRED PNEUMONIA}

${ }^{1} \mathrm{AC}$ Round, ${ }^{2} \mathrm{KF} \mathrm{Ng},{ }^{2} \mathrm{D}$ Vasudeva, ${ }^{2} \mathrm{~N}$ Manzoor, ${ }^{2} \mathrm{R}$ Radcliffe, ${ }^{2} \mathrm{D}$ Lo, ${ }^{3} \mathrm{~S}$ Koo. ${ }^{1}$ Leicester Medical School, University of Leicester, Leicester, UK; ${ }^{2}$ Paediatrics, Leicester Royal Infirmary, Leicester, UK; ${ }^{3}$ Microbiology, Leicester Royal Infirmary, Leicester, UK

\subsection{6/archdischild-2020-rcpch.260}

Aims To audit the antibiotic choice, route of administration, and duration of treatment; as well as frequency of, and indication for, chest radiographs among children admitted for CAP.

Methods A retrospective audit was conducted on patients aged 0-18 who were admitted with a diagnosis of CAP between December 2018 and February 2019. Patients were identified by International Statistical Classification of Diseases and Related Health Problems (ICD) discharge coding. Data were collected from 50 patients' case notes and electronic notes.

Results

Chest x-ray: The median number of CXR performed was 1. Performance of more than one CXR occurred in 32\% (16/ $50)$ of children, $31 \%(5 / 16)$ of whom did not require oxygen supplementation or PICU admission.

Antibiotic usage:

Antibiotic choice: Amoxicillin was prescribed as the first line antibiotic for $13 \%(6 / 48)$ of children admitted with CAP. Instead, 56\% (27/50) were started on cefuroxime, 10\% (5/48) co-amoxiclav, 8\% (4/48) ceftriaxone and 13\% (6/48) on other antibiotics. A combination of 2 or more antibiotics were given to $55 \%(26 / 48)$ of patients; $19 \%$ of which had no documented reason found. The decision to prescribe multiple antibiotics was not associated with age, presence of co-morbidity, oxygen requirement, PICU admission, length of hospitalisation or antibiotic duration.

Antibiotic route: IV antibiotics were started in $81 \%(39 / 48)$ of children, with no documented justification of this decision in 26\% (10/39) of cases. The presence of co-morbidity and oxygen requirement were not statistically associated with antibiotic route of administration.

Antibiotic duration: The median duration of antibiotics was 7 days (range 1-67 days).

Conclusions Our audit suggests there was excessive usage of broad-spectrum antibiotics for the treatment of inpatient paediatric CAP; contrary to BTS standards recommending amoxicillin as first line antibiotic choice. Following BTS standards recommending that oral antibiotics are effective even in severe CAP and the lack of association between oxygen requirement and antibiotic route, we suspect that the proportion of children started on oral antibiotic for CAP should have been higher. There is a need to improve CAP management in children, particularly regarding antibiotic choice and route of administration as well as reducing the number of unnecessary CXR performed on children admitted for CAP. 